Cahiers $d u$ MONDE RUSSE

\section{Cahiers du monde russe}

Russie - Empire russe - Union soviétique et États indépendants

$42 / 1 \mid 2001$

Varia

\title{
La réforme des institutions politiques centrales de la Russie : les «Rêveries » d'Antoine-Henri Jomini (1861).
}

\section{Ami-Jacques RAPIN}

\section{(2) OpenEdition}

1 Journals

Édition électronique

URL : https://journals.openedition.org/monderusse/8440

DOI : 10.4000/monderusse. 8440

ISSN : $1777-5388$

Éditeur

Éditions de l'EHESS

Édition imprimée

Date de publication : 1 janvier 2001

Pagination : 115-138

ISBN : 2-7132-1388-6

ISSN : $1252-6576$

Référence électronique

Ami-Jacques RAPIN, "La réforme des institutions politiques centrales de la Russie : les "Rêveries » d'Antoine-Henri Jomini (1861). », Cahiers du monde russe [En ligne], 42/1 | 2001, mis en ligne le 01

janvier 2007, consulté le 03 septembre 2022. URL : http://journals.openedition.org/monderusse/8440 ; DOI : https://doi.org/10.4000/monderusse.8440 
chercher : repérer : avancer

Cet article est disponible en ligne à l'adresse :

http://www.cairn.info/article.php?ID REVUE=CMR\&ID NUMPUBLIE=CMR 421\&ID ARTICLE=CMR 4210115

\title{
La réforme des institutions politiques centrales de la Russie : les « Rêveries » d'Antoine-Henri Jomini (1861)
}

par Ami-Jacques RAPIN

\section{| Editions de l'EHESS | Cahiers du monde russe}

\author{
$2001 / 1$ - Vol 42 \\ ISSN 1252-6576 | ISBN 2713213886 | pages 115 à 138
}

Pour citer cet article :

—RAPIN A.-J., La réforme des institutions politiques centrales de la Russie : les « Rêveries » d'Antoine-Henri Jomini (1861), Cahiers du monde russe 2001/ 1, Vol 42, p. 115-138.

Distribution électronique Cairn pour les Editions de l'EHESS.

(C) Editions de l'EHESS. Tous droits réservés pour tous pays.

La reproduction ou représentation de cet article, notamment par photocopie, n'est autorisée que dans les limites des conditions générales d'utilisation du site ou, le cas échéant, des conditions générales de la licence souscrite par votre établissement. Toute autre reproduction ou représentation, en tout ou partie, sous quelque forme et de quelque manière que ce soit, est interdite sauf accord préalable et écrit de l'éditeur, en dehors des cas prévus par la législation en vigueur en France. Il est précisé que son stockage dans une base de données est également interdit. 


\section{DOCUMENTS}

\section{LA RÉFORME DES INSTITUTIONS POLITIQUES CENTRALES DE LA RUSSIE : LES « RÊVERIES » D’ANTOINE-HENRI JOMINI (1861)}

Parmi les Suisses entrés au service de la Russie à la fin du XVIII ${ }^{\mathrm{e}}$ siècle et au début du XIX ${ }^{\mathrm{e}}$, deux d'entre eux se sont plus particulièrement prononcés sur la réforme des institutions politiques de l'empire. L'influence intellectuelle de Frédéric-César Laharpe sur Alexandre Ire est notoire : aucun manuel d'histoire n'omet de mentionner les discussions qui s'engagent entre les deux hommes au sujet des projets de transformations de la Russie à la suite de l'avènement du tsar. En revanche, l'intérêt d'Antoine-Henri Jomini pour la question est nettement moins documenté. Conseiller militaire de la cour de Russie dès 1813, Jomini est avant tout connu pour ses études stratégiques et ses réflexions relatives à l'organisation militaire de la Russie. L'Encyclopédie militaire soviétique le présente comme l'un des fondateurs de l'Académie militaire et comme le principal planificateur des opérations de la guerre contre la Turquie (1828-1829) et de la guerre de Crimée (1853-1856) ${ }^{1}$. Au-delà de l'histoire de la Russie, la réduction de la pensée de l'auteur du Précis de l'art de la guerre à sa seule dimension militaire est également de rigueur dans la littérature spécialisée. Les commentateurs de l'œuvre du général suisse négligent ainsi l'une de ses facettes qui relève de la philosophie politique. La présente publication d'un mémoire de novembre 1861, remis par Jomini à Alexandre II, permet de jeter un éclairage original sur cet aspect méconnu des conceptions du personnage. Symétrie significative, le dernier des travaux produits par le stratégiste suisse clôt un parcours intel-

1. Sovetskaja voennaja enciklopedija, Moscou, 1977, vol. III, p. 344. Le rédacteur de la notice exagère l'influence de Jomini sur la conduite des campagnes de 1828-1829 et 1853-1856. Consulté en tant qu'expert militaire, le général suisse ne joue qu'un rôle secondaire dans l'organisation des opérations. 
lectuel entamé en 1800 par la rédaction d'un projet de constitution pour la République helvétique ${ }^{2}$. Dans cet intervalle de soixante ans, ses idées politiques ont sensiblement évolué, mais son intérêt pour la « science du gouvernement » est demeuré constant. Pour bien gouverner, encore faut-il que l'autorité politique puisse s'appuyer sur des institutions qui garantissent à la fois la légitimité du pouvoir et l'efficacité de l'action étatique. Le mémoire de 1861 cherche à concilier ces deux facteurs en proposant une série de réformes visant à corriger l'organisation de l'État russe dans une phase de transformation de l'empire ${ }^{3}$.

\section{Du service de la France à celui de la Russie}

Les premiers rapports de Jomini avec la Russie remontent à l'année 1803, lorsqu'il se présente au chargé d'affaires russe à Paris pour lui remettre le manuscrit de son premier ouvrage accompagné d'une lettre destinée à Alexandre Irer. Le jeune suisse, âgé de 24 ans, cherche à se faire engager dans une grande armée européenne, et c'est sans complexe qu'il sollicite du tsar une position de lieutenant-colonel dans son armée ${ }^{4}$. Sèchement éconduit par le diplomate russe, Jomini rencontre plus de succès auprès du maréchal Ney qui l'attache en 1805 à son état-major en tant qu'engagé volontaire. Dès lors, il participe aux campagnes napoléoniennes jusqu'au lendemain de l'armistice de Pleiswitz qui suit la bataille de Bautzen (20-21 mai 1813).

Entre-temps, les qualités de l'écrivain militaire se sont imposées à l'échelle européenne. Son Traité de grande tactique (1805), puis la première édition de son Traité des grandes opérations militaires (1807-1809), suscitent un vif intérêt en raison des analyses des nouveaux principes stratégiques qu'ils contiennent. En ces circonstances, l'attitude condescendante de 1803 n'est plus d'actualité pour les agents russes qui s'efforcent au contraire d'attacher Jomini au service de leur pays. Selon les souvenirs du général suisse, des approches en ce sens sont effectuées dès 1807 par le comte Nesselrode et le prince Volkonskij5. Mais c'est en 1810 que les démarches pressantes d'Aleksandr Černišev sont le plus près d'aboutir ${ }^{6}$.

2. Le projet de constitution rédigé en 1800 nous est connu par les souvenirs de Jomini qui en présentent un exposé détaillé. Soumis à Johann Rudolf Dolder, membre du Directoire de la République helvétique, il valut à son auteur de grands éloges, mais Dolder n'y donna aucune suite. A.-H. Jomini, «Recueil de souvenirs pour mes enfants » (cité infra: "Recueil de souvenirs »), pp. 25-26, Eidgenössische Militärbibliothek (Berne), TAP 0195.

3. Ce document est conservé à la Bibliothèque militaire fédérale de Berne, en annexe au «Recueil de souvenirs » inédits de Jomini : «Rêveries sur l'état actuel de la Russie et sur l'avenir qui paraît devoir résulter de l'émancipation » (cité infra « Rêveries »), novembre 1861, Eidgenössische Militärbibliothek (Berne), TAP 0196.

4. «Recueil de souvenirs », p. 31. À cette date, Jomini pouvait se prévaloir d'une expérience d'organisation militaire au Bureau de la guerre de la République helvétique, qui lui avait permis d'obtenir le grade de chef de bataillon.

5. Ibid., p. 268.

6. Dans une lettre du 6 juin 1810 au chancelier Romanzov, le fameux agent d'influence russe à Paris explicite l'importance que présente « l'acquisition » de Jomini par son pays : « Monsieur de Jomini étant reconnu pour être un des militaires les plus instruits de la France, j'ai pensé que 
La présentation des conditions rocambolesques de cette véritable entreprise de débauchage ne relève pas de cet article : pour de plus amples détails, on se rapportera aux récits complaisants des biographes de Jomini et à celui nettement plus critique de Frédéric Masson ${ }^{7}$. Il suffit de dire ici que le projet avorte in extremis, nonobstant les dispositions favorables d'un Jomini insatisfait de l'évolution de sa carrière dans l'armée napoléonienne. Trois ans plus tard, cette même frustration le décide à abandonner les rangs français et à entrer au service du tsar en pleine campagne d'Allemagne. L'épisode du 14 août 1813 a fait couler beaucoup d'encre. La décision du transfuge a été fréquemment stigmatisée, en particulier par ses compatriotes qui y voient une grave infraction à la discipline militaire ${ }^{8}$. Pour mon propos, il n'importe pas de revenir sur les jugements portés sur cet événement. En revanche, la réaction qu'il suscite chez Frédéric-César Laharpe mérite d'être relevée. Quelques semaines après l'entrée de Jomini au service d'Alexandre Irer, son ancien précepteur s'adresse à ce dernier pour le mettre en garde contre son nouvel aide de camp 9 .

Le portrait peu flatteur que Laharpe trace de son compatriote à cette occasion at-il exercé une influence sur le tsar? Après un accueil très favorable, Jomini note que ses mérites ne sont pas reconnus à leur juste valeur et évoque même « une sorte de disgrâce » au moment du congrès de Vérone $(1822)^{10}$. Plus qu'une hostilité

l'acquisition d'un homme qui a profondément pensé et médité les grands principes de la guerre, qui les a développés et appliqués, pouvait être extrêmement précieuse pour le service de S.M. Une considération qui me paraît pas moins forte que les précédentes, c'est la connaissance intime et étudiée qu'il a de tous les individus qui figurent aujourd'hui dans les armées de l'Europe ; cet avantage inappréciable assure à la personne qui le possède une supériorité sur toute autre qui aurait égalité de génie militaire ; connaître le système, les talents ou la nullité d'un homme que l'on doit combattre, c'est déjà la moitié de la victoire. » Černišev au chancelier Romanzov, Paris, 6 juin 1810, cité in Jost Soom, « Avancement et fortune » : Schweizer und ihre Nachkommen als Offiziere, Diplomaten und Hofbeamte im Dienste des Zarenreiches, Zürich, Verlag Hans Rohr, 1996, p. 197

7. Jean-François Baqué, L'homme qui devinait Napoléon : Jomini, Paris, Perrin, 1994 ; Xavier de Courville, Jomini ou le devin de Napoléon, Lausanne, Centre d'Histoire Reprints, 1981 Ferdinand Lecomte, Le Général Jomini : sa vie et ses écrits. Esquisse biographique et stratégique, Lausanne, B. Benda, 1888 ; Frédéric Masson, Jadis et aujourd'hui, Paris, Société d'éditions littéraires et artistiques, 1908.

8. Ferdinand Lecomte, « Ma première entrevue avec le général Jomini », La Revue du Dimanche, 11, 1891, pp. 83-86 ; «Concours du Drapeau suisse sur le général Jomini. Rapport du jury », Le Drapeau suisse, 25.04.1912 ; Robert Frick, «Jomini stratège », in Le général Antoine-Henri Jomini (1779-1869). Contributions à sa biographie, Lausanne, Imprimeries réunies, 1969, p. 70.

9. «Les gazettes annoncent que le général Jomini vient de passer à votre service avec le grade de lieutenant-général. Cet homme a du talent, beaucoup de facilité, mais une présomption excessive. Mon devoir, Sire, est de vous avertir que sa moralité n'est pas vantée dans son pays. On lui reproche, en particulier, les démarches coupables que, dans le but de se recommander, il fit il y a quelques années, auprès du gouvernement français, pour l'engager à incorporer notre canton et quelques autres. Par ces motifs divers, je lui avais fait refuser ma porte. C'est un homme à surveiller de très près. » Laharpe à Alexandre I ${ }^{\text {er }}, 14$ septembre 1813, Bibliothèque cantonale et universitaire de Dorigny, Lausanne, Fonds Laharpe, IS 1918, G Aa 95. Laharpe fait ici allusion à une pétition d'avril 1804 adressée à Bonaparte. Rétrospectivement, Jomini juge son initiative de 1804 comme une « bêtise » et une « faute ». Jomini à Lecomte, 6 janvier 1863, Archives cantonales vaudoises, Lausanne, Fonds Lecomte (actuellement en classement).

10. « Recueil de souvenirs », p. 515 
marquée du monarque, il semble que la susceptibilité du général suisse et ses attentes déçues sont principalement en cause. Expert militaire parmi d'autres au sein de l'état-major coalisé lors de la campagne de 1813, ses avis ne sont pas toujours entendus et ses compétences ne sont pas récompensées à la hauteur qu'il leur accorde. Plus grave à ses yeux, les promesses de fortune formulées par Alexandre ne se réalisent pas dans les années qui suivent. Après le congrès de Vienne auquel il participe dans la suite du tsar, Jomini effectue un premier séjour de sept mois à Saint-Pétersbourg en 1815-1816. Sur demande de l'empereur, il rédige deux mémoires stratégiques - l'un sur la politique de défense de la Russie et l'autre sur le système de forteresses nécessaire pour couvrir les frontières occidentales de l'empire - initie le grand-duc Michel « aux secrets de la grande guerre » et dirige la publication d'une histoire de la campagne de 1813. Jomini quitte ensuite la Russie pour un long séjour parisien de six ans que Daniel Reichel qualifie «d'exil volontaire $»^{11}$. Si officiellement le général suisse gagne la France pour des raisons de santé, et qu'il consacre les années 1817-1823 à la rédaction de sa monumentale Histoire des guerres de la Révolution en quinze volumes, il ne fait guère de doute que cet éloignement de la cour de Russie résulte d'un refroidissement de ses relations avec le tsar.

De retour à Saint-Pétersbourg en 1824, Jomini a l'occasion de s'entretenir à plusieurs reprises avec Alexandre Irer, entretiens sur lesquels je reviendrai par la suite. Mais ce n'est qu'avec l'avènement de Nicolas Irr que s'opère un retour en grâce de l'aide de camp qui est confirmé par le nouvel empereur. En 1826, Jomini participe au comité de réforme des «institutions militaires pour la jeunesse » (écoles des cadets), puis quelques mois plus tard il est chargé de rédiger le projet d'une Académie militaire ${ }^{12}$, dont la direction lui échappe en 1832, nous dit Jomini, en raison des intrigues de Černišev ${ }^{13}$. C'est incontestablement sous le règne de Nicolas Ier que le général suisse déploie la plus grande activité professionnelle au service de la Russie. Comme mentionné précédemment, il conseille le tsar lors de la campagne contre la Turquie de 1828 et se prononce également sur les opérations de la guerre de Crimée en $1854{ }^{14}$. Entre-temps, il dirige les études du grand-duc héritier et tire de son enseignement la plus fameuse de ses études, le Précis de l'art de la guerre. Plus généralement, il produit une série de mémoires et de notes sur la politique militaire et la défense de l'empire, tous demeurés inédits. Sous le règne de Nicolas I ${ }^{\text {er }}$, ses séjours en Russie alternent avec des périodes de résidence prolongée en Belgique et en France, qu'il consacre à ses propres travaux d'écrivain militaire. Jomini quitte définitivement la Russie en 1855, année du couronnement de son

11. Daniel Reichel, «La position du général Jomini en tant qu'expert militaire à la cour de Russie », Actes du Symposium 1982, Pully, Centre d'histoire, 1982, p. 63.

12. Carl Van Dyke, Russian imperial military doctrine and education, 1832-1914, New York, Greenwood Press, 1990, p. 1.

13. « Recueil de souvenirs », pp. 573-574.

14. En 1829, il rédige une brochure «semi-officielle » intitulée Observations sur la dernière campagne de Turquie. En ce qui concerne la guerre de Crimée, il participe à des conseils de guerre, mais n'est pas associé aux opérations sur le terrain. 
ancien élève. Si Alexandre II le confirme dans ses fonctions d'aide de camp, le vieux général suisse, alors âgé de 76 ans, ne joue plus de rôle significatif dans la politique militaire de l'empire, en dehors des quelques conseils qu'il prodigue encore au printemps 1855 sur les opérations de Crimée. Le mémoire de 1861 sur la réforme des institutions politiques centrales de l'empire est donc le dernier coup d'archet d'un vieillard retiré depuis plusieurs années de la vie publique de Russie.

\section{Jomini et les affaires intérieures de Russie}

Comme on peut le constater, les attributions de Jomini à la cour de Russie relèvent du domaine exclusivement militaire. La restriction de son influence à la sphère de ses compétences professionnelles ne l'empêche pas de se prononcer de manière informelle sur des objets afférents à la politique intérieure russe. Les anecdotes rapportées à ce sujet dans ses souvenirs permettent de cerner à la fois son degré de familiarité avec la vie de l'empire et sa capacité à se positionner dans la société russe.

La première d'entre elles se rapporte à la politique financière. Lors d'un dîner intime offert par Alexandre Ier, vraisemblablement en 1816, Jomini critique l'action du ministère des Finances dans une situation de pénurie du papier monnaie qui est tombé à $25 \%$ de sa valeur. Or le comte Nesselrode assiste au dîner et l'aide de camp impérial ignore qu'il est l'époux de la fille du comte Gur'ev, ministre des Finances. Jomini conclut de sa maladresse : «Hélas, je ne me doutais guère dans ma naïveté que je me faisais là deux ennemis pour un. $»^{15}$

Quelque temps plus tard, Jomini pense avoir découvert le moyen de concilier ses intérêts personnels à ceux de l'empire, en proposant à Alexandre Ier de lui accorder le privilège d'établir à Saint-Pétersbourg une compagnie d'assurances contre l'incendie. De la sorte, sa fortune serait assurée et les sommes considérables qui sortaient de Russie au bénéfice des compagnies de Londres ou de Hambourg resteraient dans le pays ${ }^{16}$. À en croire le « Recueil de souvenirs », le tsar accueille favorablement sa requête, mais le projet d'oukase qu'il demande sur cet objet reçoit un préavis négatif du ministre des Finances qui n'est autre que le comte Gur'ev.

Ces deux anecdotes se situent lors du premier séjour de sept mois du général suisse en Russie. Il en ressort une méconnaissance manifeste de la société russe dont Jomini ignore tout, et en particulier la langue. Se remémorant ces circonstances, il souligne sa compréhension superficielle de son pays d'accueil et commente : «si l'on veut pénétrer les arcanes de l'administration, de la justice, de la machine gouvernementale, non seulement de la capitale, mais encore dans les provinces alors on peut passer des années sans se flatter de les connaître à fond $»^{17}$. Le mémoire de 1861 manifeste cette ignorance dans son préambule, lorsque l'auteur affirme ne pas suffisamment maîtriser les mécanismes administratifs et

15. « Recueil de souvenirs »,p. 497.

16. Ibid., p. 500.

17. Ibid., p. 520. 
judiciaires des gouvernements de province pour se prononcer sur les réformes au plan local (infra). Si les «Rêveries » de Jomini se limitent à discuter les réformes des institutions politiques centrales de la Russie, il faut y voir autant un attrait prononcé de l'auteur pour les questions de philosophie politique, que son incapacité à aborder le détail de l'administration de l'empire.

En 1824, le second séjour de Jomini en Russie est explicitement destiné à l' " initier dans les mystères de la société [russe] ». À cette occasion, il rencontre les principaux personnages de la haute société et de la fonction publique, en particulier Mihail Speranskij. Le portrait qu'il trace du « législateur libéral » nous livre les premières considérations de Jomini sur le fonctionnement de la machine gouvernementale russe :

« J'avais beaucoup entendu parler du fameux Speransky, dont on a tour à tour exagéré et décrié le mérite, j'allai le voir ainsi que l'historien Karamsin [Nikolaj Karamzin], et tous deux me parurent des hommes capables de jouer un rôle dans une machine gouvernementale dont les ressorts offriraient plus d'harmonie et plus d'élasticité. Mais dans un pays où tout se résume par un rapport (doklad) à l'Empereur, où aucun fonctionnaire n'a ni initiative ni compétence dans certaines limites bien tracées par de bons règlements, il est bien difficile que de grands ministres se produisent. Un Pitt ou un Colbert resteraient dans l'ombre, là où il n'y a qu'un favori ou une camarilla qui gouvernent, quand le maître n'est pas un grand homme. $~^{18}$

Au dire de Jomini, cette vision d'une bureaucratie lourde et inefficace est partagée par Alexandre Irer. Répondant à une question du monarque sur les moyens de diminuer la « paperasserie », l'aide de camp propose de nommer six ministres capables et probes, de leur tracer de larges compétences, mais subordonnées à des règlements irrévocables et sagement rédigés, et enfin de punir sans pitié les malversations et les abus de pouvoir ${ }^{19}$. L'esprit du mémoire de 1861 est résumé dans cette recommandation de 1824. Pour Jomini, le monarque doit assurer la pérennité de son autorité en transformant les mécanismes du pouvoir autocratique. Le règne de l'arbitraire et les abus de pouvoir sapent les fondements de l'empire qui ne pourra retrouver son assise politique qu'au prix d'une réforme de ses principes de gouvernement. À l'exemple de Montesquieu - seul penseur politique cité dans le mémoire - Jomini voit dans l'existence de lois fixes et établies le plus sûr rempart contre le règne de l'arbitraire. Toujours en accord avec l'auteur de L'Esprit des lois, le mémoire de 1861 cherche à instaurer des «pouvoirs intermédiaires » entre la volonté du monarque et la masse de ses sujets, des « canaux moyens par où coule la puissance $»^{20}$ et qui garantissent le bon usage des lois.

Mais la réforme des rouages étatiques ne représente qu'une facette du problème de la modernisation de l'empire. À l'exemple de Laharpe, et de beaucoup d'autres,

18. Ibid., pp. 521-522.

19. Toujours selon Jomini, le tsar lui aurait rétorqué : « Ah ! général, croyez-vous donc que six hommes à la fois probes et capables soient si faciles à trouver... », ibid., p. 522.

20. Les deux formules figurent dans le chapitre 4 du livre II de L'Esprit des lois. Si Jomini ne les utilise pas explicitement, elles me semblent toutefois parfaitement rendre compte du sens des réformes proposées dans son mémoire. 
Jomini est favorable à l'abolition du servage. Chez le général suisse, les motifs philanthropiques et philosophiques vont de pair avec une volonté de promouvoir le bien de l'État et d'assurer le développement économique du pays. En 1824, il confie à Alexandre Irer ses vues sur la question. Dans l'hypothèse d'une émancipation, les paysans libérés du servage seraient appelés à former un «tiers-état respectable » de bourgeois, de marchands et d'ouvriers accroissant la population urbaine et créant un marché de consommation pour la production de l'empire. Cette augmentation des « classes moyennes » favoriserait à son tour le « développement graduel des Arts et de l'industrie qui certes réagirait sur la prospérité de l'État et sur sa puissance ». Toujours selon Jomini, ses considérations entraînent la réponse suivante d'Alexandre Irer :

«Tout cela peut être parfaitement juste, mais l'exécution offre des obstacles dont on ne saurait se rendre compte sans connaître à fond les rapports des serfs avec leurs seigneurs. Il n'y aura que le temps qui pourra résoudre cette question, en attendant étudiez-la sous toutes ses faces et soyez réservé. $\gg^{21}$

L'interdépendance des deux types de réformes nécessaires à la modernisation de l'empire est clairement identifiée par Jomini. Toutefois, comme Laharpe, il ne semble pas nettement distinguer les procédés concrets susceptibles de les réaliser. Il faut attendre le manifeste d'Alexandre II de février 1861 pour que Jomini propose un projet explicite de transformation des institutions politiques de l'empire. Dans l'intervalle, le « Recueil de souvenirs » est silencieux sur ce sujet ; l'auteur indique simplement qu'il soutint fréquement ses positions dans des conversations avec quelques-uns de ses amis, en particulier le maréchal Ivan Paskevič et le général Dimitrij Buturlin²2. En revanche, l'aide de camp de Nicolas Ier ne paraît pas avoir fait part de ses convictions à l'autocrate.

\section{Le projet de Jomini}

Le projet de réforme du général suisse vise à refondre les institutions centrales de la Russie dans une perspective d'efficacité et de gouvernabilité. Ce faisant, il est confronté au même problème sur lequel ont buté toutes les tentatives de réformes précédentes : comment améliorer l'exercice du gouvernement sans battre en brèche le principe autocratique ? La réponse de Jomini s'éloigne des desseins immédiatement constitutionnels, en formulant un compromis entre la monarchie absolue et un régime d'assemblées délibérantes. L'auteur est conscient qu'une séparation purement fonctionnelle des pouvoirs ne résout qu'imparfaitement le problème de gouvernement du pays. Son compromis se doit donc d'accorder une place à la nation dans les institutions étatiques, tout en préservant celles-ci du danger que représente à ses yeux un régime parlementaire. En conséquence, il ne saurait être

21. « Recueil de souvenirs », p. 525.

22. Ibid., p. 660 . 
question ni d'un authentique équilibre des pouvoirs entre les chambres législatives et l'exécutif, ni bien sûr d'une démocratie représentative. Si le projet comporte une timide ouverture politique vers l'évolution du régime dans un sens représentatif, celle-ci demeure entièrement tributaire de la volonté du monarque. À cet égard, le mémoire du général suisse exprime autant ses convictions sur la forme spécifique que doit prendre l'exercice du pouvoir en Russie que les éléments d'une philosophie conservatrice forgée sur la base d'une critique des institutions politiques de la France de la Restauration.

La genèse du projet que Jomini présente à Alexandre II sous le nom de « Rêveries sur l'état actuel de la Russie et sur l'avenir qui paraît devoir résulter de l'émancipation » s'inscrit dans le prolongement du manifeste du 19 février 1861. Ce dernier soulevait à la fois un obstacle à la réforme des institutions politiques de l'empire et la rendait aussi indispensable qu'urgente. Les conséquences politiques de l'émancipation sont vraisemblablement méditées par Jomini au cours de l'année. Ses réflexions sur le sujet donnent lieu à la rédaction de plusieurs esquisses, puis, en novembre 1861, Jomini finalise son travail dans un mémoire d'une dizaine de pages. Initialement, son intention était de le soumettre à l'ambassadeur de Russie à Paris. Toutefois, le peu d'empressement de ce dernier pousse Jomini à transmettre le mémoire à son fils Alexandre, conseiller privé au département des Affaires étrangères à Saint-Pétersbourg, qui consulte son ministre, le prince Gorčakov, puis transmet le document au tsar.

Alexandre II prend connaissance de l'original - le fils de Jomini « jugea bon de l'envoyer à l'Empereur sans faire recopier le manuscrit $\gg^{23}-$ et c'est ce même manuscrit qu'il retourne à l'auteur, un mois plus tard, accompagné de quelques notes marginales et de ses remerciements. L'original annoté a vraisemblablement été perdu par Jomini qui précise, dans une brève note introductive, que seules les premières minutes du document «qui diffèrent fort peu de la rédaction finale soumise à l'Empereur » ont été conservées ${ }^{24}$.

L'intitulé du mémoire pose la question des intentions de son auteur. Faut-il voir dans l'utilisation du terme de « rêveries » une référence à une lecture de jeunesse de l'écrivain militaire, les Rêveries du maréchal de Saxe ? Peut-être. Mais ce choix renvoie plus sûrement à l'ambivalence de la démarche jominienne. Ce titre semble dénoter des considérations informelles, ce qui est manifestement contredit par la rédaction finale d'une formule d'oukase qui en dit long sur la valeur que l'auteur accorde à son propos. Âgé de plus de quatre-vingts ans, le vieux général précise qu'il remplit un «devoir sacré » et que ce document qui «fut le dernier de mes travaux, n'en fut pas le moins bon $»^{25}$.

Le mémoire se compose de quatre parties distinctes. Un préambule sommaire examine les réformes de l'administration aux niveaux local et régional. Ensuite, deux hypothèses sont formulées en vue de transformer les instruments du pouvoir

23. Ibid., p. 664.

24. « Rêveries».

25. « Recueil de souvenirs », pp. 540 et 664. 
central. La première envisage un gouvernement autocratique. La seconde examine l'organisation d'un régime monarchique tempéré par des lois fondamentales. Enfin, la formule d'un oukase formalise la seconde hypothèse vers laquelle va la préférence de l'auteur.

La brièveté du préambule résulte d'une cause évoquée précédemment. Jomini ne maîtrise pas suffisamment les mécanismes des pouvoirs locaux pour se prononcer sur le détail de leur réforme. Par ailleurs, il conclut justement que cette entreprise ne peut relever que de commissions spécialisées désignées par le gouvernement central. La réforme de ce dernier apparaît donc comme un préalable à une gestion efficace de l'ensemble des transformations induites par l'émancipation. Malgré la restriction apportée à son propos, Jomini identifie deux secteurs clés des réformes au niveau local. L'un relève du domaine organisationnel et touche la fiscalité et l'administration de la justice. L'autre concerne le domaine législatif. À ce propos, Jomini insiste sur la nécessité de poursuivre l'effort de codification entrepris dans les années 1830 sous le règne de Nicolas Ier $:$ le recueil des lois de l'empire est trop massif, sa législation trop « embrouillée », il convient donc de la réduire à cinq ou six codes d'un seul volume chacun. Si Jomini insiste sur ce dernier point, c'est qu'une législation maniable est une condition sine qua non au bon fonctionnement des instruments de gouvernement, tant sur le plan local que sur le plan de l'État central.

En matière d'institutions centrales, Jomini partage l'un des lieux communs de la pensée politique russe : les dimensions de l'État exigent un pouvoir central fort qui s'incarne en une personne, un axiome qui a pour corollaire que toute réforme ne peut être décidée qu'au sommet de l'État. Chez le général suisse, cette conviction est renforcée par deux types de considérations plus personnelles exposées dans un ouvrage de 1839 livrant les éléments de sa philosophie politique. Les premières sont fondées sur une vision pessimiste des relations internationales :

«Les grandes sociétés européennes, quoi qu'en disent tous les Don Quichotte de la métaphysique gouvernementale, ne seront jamais que des sociétés égoïstes et rivales les unes des autres. Dès lors, toute société bien ordonnée doit investir ses chefs de toute la force indispensable pour les rendre redoutables au dehors et respectés à l'intérieur. $»^{26}$

Dans cette perspective, l'existence d'un principe d'autorité fortement constitué est non seulement une nécessité propre à l'exercice du pouvoir en Russie, mais aussi une condition à la stabilité politique de l'Europe. Les secondes considérations de Jomini résultent de son analyse des institutions politiques de la France, et plus particulièrement de celle de la Charte de 1814 octroyée par Louis XVIII, un document auquel il est fait référence dans le mémoire. Une lecture parallèle du mémoire de 1861 et de l'ouvrage de 1839 permet d'établir la similitude des conceptions politiques qu'ils contiennent, même si Jomini précisait à la fin des années 1830 que ses

26. A.-H. Jomini, Précis politique et militaire de la campagne de 1815 , pour servir de supplément et de rectification à la Vie politique et militaire de Napoléon, Paris, Anselin et Laguyonie, 1839 , pp. 253-254. 
jugements ne sauraient s'appliquer à la Russie ${ }^{27}$. À l'époque, le règne autocratique de Nicolas Ir ne lui permettait pas de se prononcer sur une éventuelle réforme politique de l'empire. Vingt-deux ans plus tard, le processus initié par le «tsar libérateur » crée les conditions d'une généralisation des principes politiques du général suisse à la Russie. À cet égard, les analyses de 1839 permettent de préciser certains points sommairement exposés dans le mémoire de 1861 et, au-delà, indiquent le sens de l'ouverture politique proposée dans ce dernier document.

Après avoir écarté l'hypothèse républicaine, qu'il juge absurde pour un grand État européen, Jomini envisage les différentes formes de l'exercice du pouvoir monarchique. Le principe d'une monarchie élective est d'emblée rejeté, dans la mesure où un tel régime est source de conflits internes, nous dit l'auteur en 1861, mais aussi d'interventions externes, précise-t-il en 1839. Seule la monarchie héréditaire lui semble donc souhaitable, principalement pour des raisons de stabilité politique. Dès lors, deux hypothèses sont prises en considération, l'une relative à une monarchie héréditaire absolue, l'autre à une monarchie héréditaire «tempérée » et non pas constitutionnelle, un terme que Jomini se garde d'utiliser. Dans les deux cas, l'auteur propose des structures institutionnelles communes, les variantes se distinguant par l'introduction d'un bicaméralisme partiellement représentatif et surtout par l'intention limitative contenue dans la seconde variante.

L'hypothèse absolutiste est brièvement examinée, dans la mesure où elle ne correspond visiblement pas à l'inclination de l'auteur. Elle reprend les éléments du système institutionnel traditionnel, mais en précise les attributions. En premier lieu, le Conseil privé appuie le jugement du monarque dans l'exercice du pouvoir. Sa composition relève du choix de l'empereur qui, dans son intérêt, doit se détourner de ses familiers pour ne sélectionner ses membres qu'en fonction de leurs compétences. En deuxième lieu, le Conseil des ministres forme l'organe central du gouvernement. Ses attributions ne sont que succinctement évoquées dans le mémoire, mais il est clair que Jomini vise à donner une unité à la marche du pouvoir en instituant une véritable politique de cabinet responsable devant l'empereur. Sur ce point, le « Recueil de souvenirs » fournit quelques précisions en indiquant que le conseil doit se réunir périodiquement sous la présidence de l'empereur ou sous celle d'un viceprésident désigné par le monarque ${ }^{28}$. En d'autres termes, la responsabilité individuelle des ministres envers l'empereur est abandonnée au profit d'une responsabilité collective et, plus important, l'hypothèse d'une délégation de la direction du conseil est envisagée ${ }^{29}$. En troisième lieu, le Conseil de l'Empire (Conseil d'État) est chargé de l'élaboration et de la rédaction des lois et des règlements.

À ce niveau de l'argumentation, les réformes proposées n'ont rien de décisif. Jomini cherche uniquement à corriger les dysfonctionnements du régime autocra-

27. Ibid., p. 13.

28. « Recueil de souvenirs », p. 663.

29. La carence d'un véritable organe central de gouvernement était suffisamment évidente pour qu'Alexandre II décrète la création d'un tel Conseil des ministres en novembre 1861, soit au moment où Jomini termine la rédaction de son mémoire. 
tique, en introduisant une division structurelle des pouvoirs entre les organes exécutifs et législatifs. Le système politique projeté est trop imparfaitement esquissé pour qu'apparaisse distinctement le sens des réformes élaborées par le général suisse. Ses idées se précisent à partir du moment où il aborde la création d'une nouvelle institution qu'il présente sous la dénomination de Conseil législatif. Néanmoins, le mémoire manque de clarté sur le statut exact de ce nouvel organe étatique. Tantôt le Conseil législatif semble relever de l'hypothèse absolutiste, tantôt il apparaît comme une composante essentielle de la forme tempérée du pouvoir monarchique. La première interprétation est étayée par la structure de l'argumentation: à deux reprises, Jomini mentionne que ses développements précédents - où il était question du Conseil législatif - se rapportent aux principes du gouvernement personnel absolu. La seconde interprétation repose sur deux énoncés significatifs : l'un établit qu'une assemblée législative élective ne saurait appartenir à la forme du pouvoir absolu, l'autre précise que la création d'une Chambre de pairs, en plus du Conseil législatif, est un « pas de plus » vers la monarchie tempérée. Cette apparente contradiction me semble se résoudre dès lors que l'on accorde sa juste portée à l'adjectif qui qualifie le Conseil législatif. Il est « mixte », non seulement dans le sens où il est partiellement électif, mais également dans la mesure où il incarne un moyen terme entre les deux formes de pouvoir discutées par l'auteur. Du point de vue argumentatif, sa présentation opère la transition entre les deux types de monarchie décrits dans le mémoire. Du point de vue institutionnel, sa création constitue une étape intermédiaire conduisant au bicaméralisme voulu par le général suisse. De ce qui précède, il résulte que la troisième partie du mémoire est partiellement imbriquée dans la deuxième, et que la défense d'une monarchie tempérée débute dès les jugements portés par Jomini sur le principe d'équilibre des pouvoirs.

Critiquant la Charte octroyée par Louis XVIII en 1814, Jomini livre ses conceptions relatives à la portion de souveraineté revenant respectivement à l'autorité royale et à la nation. Si le trône est la « propriété légitime » de la dynastie, le droit de prendre part à la confection des lois est une "propriété imprescriptible de la nation $»^{30}$. En présence d'un héritier légitime, la nation ne peut disposer du trône, mais le trône ne peut nier à la nation une part de la gestion des affaires de l'État. Toutefois, une double restriction intervient pour diminuer la portée des droits démocratiques. Premièrement, la représentation nationale ne concerne que les classes supérieures et moyennes, à l'exclusion des «masses populaires » qui sont «par leur nature appelées à être dirigées et non pas à diriger $»^{31}$. Secondement, l'initiative des lois ne revient pas aux chambres, dont les prérogatives se limitent à discuter les projets de lois et à proposer des amendements. Dès lors que l'équilibre des pouvoirs crée les conditions d'une restriction de l'autorité exécutive, l'auteur le juge néfaste à l'action « une et indivisible » du gouvernement. La crainte d'un effacement du monarque au profit d'une responsabilité politique du gouvernement, et

30. A.-H. Jomini, Précis politique et militaire..., op. cit., p. 26.

31. Ibid., pp. 101-102. 
de son contrôle par le législatif, justifie cette dernière considération qui vise à éviter une évolution des institutions politiques vers un système parlementaire à l'anglaise.

Sur cette base restrictive, Jomini développe ses conceptions d'une monarchie tempérée, telle qu'Alexandre II pourrait l'instaurer dans son empire. Aucun changement n'intervient aux niveaux du Conseil des ministres et du Conseil privé par rapport à l'hypothèse absolutiste, si ce n'est que le second de ces conseils n'est pas jugé indispensable. Quant au Conseil d'État, sa composition est précisée par l'énumération des sections qui le constituent. Jomini poursuit en présentant les caractéristiques d'une nouvelle institution, dont il avait souligné l'importance dans sa discussion de l'hypothèse absolutiste. Il propose la création d'un Conseil législatif «mixte », dans le sens où une partie de ses 216 membres sont nommés par l'empereur et une autre élue par la noblesse et les classes moyennes. Sur ce point, le mémoire comporte une ambiguïté, puisqu'il mentionne dans un premier temps que les 54 conseillers des classes moyennes sont « choisis », puis dans un second temps qu'ils sont «élus » par les bourgeois des villes. L'intention de Jomini va indubitablement vers cette seconde proposition et il faut considérer l'utilisation du premier de ces verbes comme une maladresse de rédaction. Cette assemblée est destinée à donner la sanction nationale aux projets de lois élaborés au Conseil d'État sur la requête du Conseil des ministres. Elle est également appelée à donner la sanction nationale aux mesures du gouvernement, en particulier en ce qui concerne le budget $^{32}$. Au demeurant, elle reste soumise à l'influence décisive du trône qui a la faculté de nommer, pour le moins, la moitié de ses membres. Ce procédé permet ainsi d'écarter « tous les dangers des assemblées délibérantes ambitieuses » (infra).

Enfin, Jomini propose la création de deux institutions spécifiques qui n'étaient pas mentionnées dans son hypothèse absolutiste. Tout d'abord, une Haute cour impériale de justice fait office de Cour de cassation et de Cour d'appel ; elle est formée par une partie des membres de l'ancien Sénat, l'autre partie étant appelée à siéger dans le Conseil d'État. Ensuite, l'auteur propose de «faire un pas de plus vers la monarchie tempérée » en instituant une Chambre de pairs, lesquels sont choisis par le souverain dans la noblesse et la haute administration. Ses prérogatives sont les mêmes que celles du Conseil législatif partiellement électif : elle ne peut délibérer que sur des projets de lois préparés par le Conseil d'État et transmis par le Conseil des ministres, elle a en outre la faculté d'y introduire des amendements ou des modifications soumis à la sanction du ministère. Un point demeure obscur quant au mécanisme d'adoption des lois par les deux chambres législatives. Si le projet d'oukase de Jomini précise, dans son point 6, qu'en cas de rejet d'une loi par l'une des deux assemblées, celle-ci peut soumettre ses observations au ministère qui dispose cependant de la faculté d'imposer cette loi avec l'accord du souverain - rien n'est dit dans l'hypothèse du refus d'une loi par les deux chambres. Tout porte à croire que les conceptions de l'auteur vont dans le sens d'un rejet de la loi qui devrait être présentée aux deux chambres sous une nouvelle formulation. Notons que ce cas de figure est toutefois improbable, dès lors qu' une chambre

32. Ce dernier point est rapidement évoqué en fin de document. 
est intégralement nommée par le souverain et que la moitié, pour le moins, de l'autre l'est également.

Les réformes proposées par Jomini sont limitées dans leurs conséquences politiques, et à ce titre demeurent en deçà d'un projet tel que celui présenté par Speranskij cinquante ans plus tôt. Ce que cherche le général suisse, c'est un compromis entre l'absolutisme et la souveraineté nationale, entre l'autocratie et un système parlementaire à l'anglaise. Dans cette perspective, la monarchie tempérée s'apparente à un régime qui fixe et borne l'intervention de la nation dans les institutions monarchiques et simultanément légitime ces dernières par des lois fondamentales. Celles-ci assurent les droits et le fonctionnement des assemblées législatives et, plus généralement, de l'ensemble des organes des pouvoirs exécutifs, administratifs et judiciaires. Les points 4 et 8 du projet d'oukase remis par Jomini à Alexandre II tendent vers l'instauration d'un tel « pacte national », dès lors que l'un stipule que tous les projets de loi seront dorénavant présentés aux deux chambres, et que l'autre établit que le Conseil d'État doit sans délai élaborer les lois organiques nécessaires au fonctionnement des assemblées. Jomini ne parle pas de constitution, mais laisse la porte ouverte à une évolution constitutionnelle du régime, pour autant que le tsar le désire. Son projet distribue les meilleures cartes politiques dans la main de l'empereur, mais il l'invite également, in fine, à les utiliser pour prolonger la timide ouverture qu'il lui propose (infra).

Quel fut l'accueil réservé à la démarche du vieillard retiré à Passy ? Ses souvenirs laissent transparaître une pointe d'amertume lorsque Jomini note que le manuscrit lui fut retourné par Alexandre II avec «quelques notes marginales assez insignifiantes » et un billet de remerciement relevant que le document contenait plusieurs propositions qui pourraient être utilisées en temps opportun ${ }^{33}$. En 1861, Jomini est trop vieux et trop éloigné des cercles dirigeants de Saint-Pétersbourg pour que son initiative soit véritablement prise en considération. Pour compatibles qu'elles fussent avec le dessein d'une transformation progressive des institutions politiques de l'empire, ses conceptions étaient appelées à rejoindre la masse des projets de réformes demeurés sans impact immédiat sur l'évolution du pouvoir en Russie. Au demeurant, elles dénotent la philosophie politique conservatrice de l'un des plus pénétrants théoriciens de la stratégie, tout comme son intérêt prolongé pour les affaires intérieures d'un pays qu'il choisit de servir durant de longues années.

Institut de recherches interdisciplinaires

Faculté des siences sociales et politiques

Université de Lausanne

CH-1015 Dorigny

e-mail : ami-jacques@gve.ch

33. « Recueil de souvenirs »,p. 664. 


\section{RÊVERIES SUR L'ÉTAT ACTUEL DE LA RUSSIE ET SUR L'AVENIR QUI PARAÎT DEVOIR RÉSULTER DE L'ÉMANCIPATION novembre $1861^{*}$}

La grande mesure de l'émancipation des paysans doit évidemment changer la face de la nation, si elle est menée à bonne fin. Elle lui imprimera nécessairement une vie nouvelle, mais elle exigera aussi une grande réforme dans son administration intérieure qui me parait devoir être réorganisée successivement sur de nouvelles bases. Je vais essayer d'en esquisser quelques-unes.

$1^{\circ}$ Les paysans, cessant d'être gouvernés par leurs seigneurs, devront être régis par des autorités administratives et judiciaires plus nombreuses.

$2^{\circ}$ L'empire étant divisé en 54 gouvernements subdivisés en un grand nombre de districts, il faudra, outre le gouverneur civil de chaque gouvernement, un sousgouverneur pour chaque district et des autorités communales lui obéissant.

$3^{\circ} \mathrm{Il}$ faudra un tribunal civil de $1^{\text {re }}$ instance par district et une cour de justice par Gouvernement, remplissant les doubles fonctions de tribunal d'appel au civil et de cour criminelle pour délits et crimes. Les autorités communales seront organisées et soumises au sous-gouverneur du district et il serait à désirer qu'il y eût des juges de paix dans les grandes communes.

$4^{\circ}$ Les assemblées de la noblesse seront maintenues, du moins provisoirement, les attributions du maréchal de la noblesse seront modifiées selon les circonstances.

$5^{\circ} \mathrm{Il}$ sera indispensable de rédiger un code simple et précis de procédure tant civile que criminelle, basée sur la publicité des débats qui auront lieu verbalement et oralement.

$6^{\circ}$ La perception des impôts exigera une nouvelle organisation des employés des finances dans chaque gouvernement.

$7^{\circ}$ Enfin, il serait urgent de procéder à la codification des 50 volumes d'oukases qui font la base de la législation si embrouillée de l'empire et qui seraient réduits à 5 ou 6 codes d'un seul volume chaque.

\footnotetext{
* Les passages soulignés le sont dans l'original. L'orthographe a été adaptée aux normes contemporaines, contrairement à la ponctuation et à l'usage des majuscules qui respectent la rédaction de l'auteur.
} 
Tels sont, en résumé, les travaux herculéens qui semblent imposés au gouvernement impérial et qui exigent une solution plus ou moins prompte. Je ne connais pas assez profondément tout le mécanisme actuel administratif et judiciaire des gouvernements de l'intérieur pour entrer dans l'examen de ce qui serait à conserver, modifier ou ajouter, c'est au Conseil de l'Empire ou à celui des ministres à y procéder avec maturité en nommant à cet effet des commissions spéciales composées d'hommes versés dans la science administrative, législative et judiciaire.

Une mesure non moins grave et plus délicate encore que celle de la réorganisation provinciale serait celle de raccorder les institutions centrales telles que le Sénat, le Conseil de l'Empire et le conseil des ministres avec les besoins futurs de la génération nouvelle. On comprend qu'il ne s'agit point ici de soulever ces questions brûlantes, qui ont agité toute l'Europe depuis 1789 et qui ont inspiré aux nations des théories plus ou moins spécieuses ou dangereuses formulées dans des Constitutions ou Chartes, minutieuses prétendant tout soumettre à une règle invariable.

Après ce que Montesquieu a écrit dans son immortel ouvrage de l'esprit des lois, après ce que j'ai écrit moi-même dans mes divers ouvrages, notamment dans mon précis historique et politique de la campagne de 1815 (examen des chartes modernes) je ne saurais rien dire de bien neuf sur les différentes formes du gouvernement monarchique. (Je ne parlerai pas des différentes espèces de républiques, elles n'ont rien à faire ici). Quant aux monarchies, elles sont de trois espèces :

$1^{\circ}$ L'autocratie ou le gouvernement absolu personnel (qui sont absolument identiques).

$2^{\circ}$ La monarchie tempérée par des lois, mais soumise à la légitimité héréditaire.

$3^{\circ}$ La monarchie élective, source de toutes les guerres intestines et de toutes les anarchies.

Le titre d'Autocrate a été de nos jours jeté à la face des princes comme un titre injurieux équivalent à celui de Tyran, de Néron, de Caligula, tandis qu'il ne désigne autre chose que le pouvoir personnel tel que les rois de France l'ont exercé jusqu'à Louis XVI et les empereurs d'Autriche ou les rois de Prusse jusqu'en 1848.

Croire qu'un monarque autocrate règne seul sur les peuples est une absurdité qui n'est que trop répandue dans les masses, même dans les classes moyennes. Il n'est pas de pouvoir plus absolu que celui des sultans de Constantinople, or on sait comme ils sont obéis hors du rayon de la capitale. Les puissants empereurs de Russie eux-mêmes ne font pas toujours prévaloir leur volonté sur toute la surface de l'empire, forcés de s'en rapporter aux agents de leur autorité, ils prennent souvent des mesures sur des rapports infidèles ou erronés, et les autorités locales ont autant de pouvoir et d'influence sous une autocratie que sous toute autre forme du gouvernement. Leurs ministres même, sous leurs yeux, se permettent souvent d'éluder leurs ordres et de substituer leur volonté à celle du maître ; j’en ai des preuves par moi-même.

En consultant les leçons de l'histoire, on reconnaîtra que lorsqu'un monarque est à la fois habile, juste et énergique, le pouvoir absolu ou l'autocratie est la forme de gouvernement la plus forte et souvent la plus efficace, celle où le chef de l'État 
pourra faire le plus de bien et corriger le plus d'abus ; mais c'est là une éventualité qui ne saurait faire règle générale, car l'on sait ce que peut devenir une monarchie absolue sous un Louis XV et même sous le trop vertueux et faible Louis XVI. D'ailleurs, en accordant la plus grande habileté au monarque, ce serait un étrange sophisme de prétendre qu'il règne uniquement par lui-même et sans l'aide d'un conseil ! Le fier Louis XIV ne publiait ses ordonnances que sous la forme « Le roi en son Conseil »; s'il rabaissa l'influence des parlements, il ne s'en passa pas entièrement et Dieu sait ce que son règne eût été sans les conseils de Colbert et de Louvois !!

De ces vérités on peut conclure que le premier intérêt d'un monarque, même le plus absolu, sera toujours de s'entourer d'un conseil privé composé d'hommes les plus capables et non des familiers ou des favoris du prince dont l'influence a été si souvent nuisible à la gloire d'un règne comme aux intérêts de l'État. Sans doute, si un ami du Prince, possédant sa confiance se trouvait doué de talents incontestables, il serait naturellement appelé dans ce conseil, mais ce serait une exception et non une règle ; l'intérêt du Prince lui-même doit le détourner de discuter les affaires de l'État avec ses familiers. Après ce conseil privé, vient le conseil des ministres ou secrétaires d'État, qui forment les représentants naturels de l'autorité royale dans les différents dicastères; institution indispensable pour donner une marche uniforme aux différents rouages de l'administration, et qui, loin de diminuer l'autorité du Prince, ne peut que l'éclairer et la seconder.

On comprend qu'indépendamment de ces deux conseils, il en faut un troisième qui élabore et rédige les lois et règlements de l'administration publique projetés par le ministère. Ces fonctions sont en partie exercées aujourd'hui par le Conseil de l'Empire, dénomination qui parait moins logique et laisse du vague dans ses attributions. Mais quelque nom qu'on lui donne, il faut se garder de confondre ce conseil avec une assemblée législative élective qui rentrerait dans la monarchie tempérée et n'appartient plus à la forme du pouvoir absolu.

À ces différents établissements on pourrait, à mon avis, en ajouter un bien important, sans atténuer en rien l'autorité royale et sans l'exposer aux inconvénients des gouvernements dits Parlementaires, ce serait la création d'un Conseil législatif chargé de discuter les projets de lois préparés par les mandataires de la Couronne et qui serait composé en partie de conseillers nommés par le monarque, en partie de députés de la noblesse et des classes moyennes.

Ne pouvant discuter que les propositions du gouvernement, n'y introduire des modifications qu'avec l'assentiment du Conseil des ministres, une pareille assemblée n'aurait [rien] de commun avec les chambres parlementaires des gouvernements constitutionnels et elle offrirait cependant l'avantage d'une discussion entre les députés des provinces et ceux nommés par l'autorité royale. Si une pareille institution mixte paraissait dérisoire aux partisans des doctrines anglaises, je la crois plus convenable dans les circonstances actuelles pour maintenir la force dans l'autorité tout en l'éclairant sur les besoins du pays ; on pourrait avec le temps étendre et perfectionner ses attributions.

Tout ce qui précède se reporte naturellement aux institutions d'un gouvernement autocratique, mais pour compléter cet aperçu on me permettra de passer 
maintenant à l'examen de la monarchie tempérée par des lois. Elle se présente sous différentes formes. La plus remarquable est certainement celle qu'on a définie en quelque sorte par cette étrange phrase : Le roi règne et ne gouverne pas. Théorie absurde que les utopistes ont emprunté aux Anglais, bien qu'aucune charte anglaise n'empêche un roi de gouverner, ainsi que Guillaume III l'a bien prouvé.

Depuis que les Anglais ont été chercher un roi étranger dans la maison de Brunswick Hanovre, le hasard a voulu que les princes placés conditionnellement sur le trône fussent des hommes médiocres, trop heureux de jouir des charmes d'une royauté nominale, ou des femmes heureuses de laisser à leurs ministres le soin de gouverner et de diriger les parlements. Mais si un homme vraiment supérieur occupait ce trône, rien ne l'empêcherait de prendre cette direction, bien entendu, en s'appuyant sur les conseillers de la nation et en les accordant avec ceux de la couronne.

Mais l'idée d'établir en principe qu'un roi doit être le roi soliveau de la fontaine, un ruineux pensionnaire de l'État, uniquement destiné à se prélasser dans ses palais et à y donner des fêtes est une des plus étranges absurdités proclamées dans ces temps de troubles et de révolution où l'on déclarait en plein parlement que les députés étaient faits pour entraver la marche de l'autorité.

Repoussant donc de pareilles doctrines, contentons-nous de parler des différents modes d'exercer l'autorité royale, sans trop atténuer sa force et sa dignité. On peut le faire par une charte fondamentale, complète et détaillée, réglant tous les rapports du monarque et des différentes autorités avec la nation et ses représentants, un pacte sacré consacrant à la fois les droits et les devoirs de tous.

On peut le faire aussi d'une manière moins absolue et moins minutieuse, se bornant à poser un petit nombre de bases immuables, les principes généraux et les droits comme les devoirs laissant à la royauté et à la législature le soin d'en développer successivement l'application, selon les événements et les besoins de l'État, évitant ainsi de renfermer le gouvernement royal et les chambres législatives dans des limites absolues qui, tôt ou tard, peuvent faire surgir des conflits.

À la tête des Constitutions, de la première catégorie, il faut placer la charte octroyée par Louis XVIII, qui semblait, en effet une œuvre de perfection offrant les garanties les plus désirables de stabilité et de force pour l'autorité et donnant aux représentants du pays une juste part d'influence dans la confection des lois et la direction des affaires. On se flattait d'avoir trouvé la pierre philosophale, l'équilibre parfait des pouvoirs.

C'était une étrange erreur comme on peut s'en assurer par le calcul suivant : une loi reconnue nécessaire était élaborée au Conseil d'État, discutée au Conseil des ministres, approuvée par le Roi, soumise aux chambres ; elle pouvait être adoptée à l'unanimité par la première et repoussée par la seconde avec quatre ou cinq voix de majorité seulement. Il résultait de là que quatre ou cinq députés ou pairs qui avaient voté contre au lieu de voter pour avaient le pouvoir d'annuler l'œuvre du Conseil d'État, des Ministres, du Roi et de la totalité d'une chambre.

Et on appelait cela un équilibre !!! 
On objectera qu'on avait imaginé la dissolution de la chambre des Députés ou des fournées de Pairs comme des moyens de corriger cette anomalie, hélas! le remède était pire que le mal, l'expérience ne l'a que trop prouvé. Pour obtenir un véritable équilibre entre trois pouvoirs, il faudrait nécessairement que la volonté de deux pouvoirs prévalût sur celle du troisième : la seule modification que l'on peut admettre pour satisfaire ce dernier, serait de lui accorder le pouvoir suspensif en exigeant que la loi repoussée ne fût représentée qu'après un nouvel examen, mais en lui donnant force de loi dans le cas où elle serait de nouveau sanctionnée par les trois autorités qui l'avaient admise, lors même que la quatrième persisterait dans son rejet.

On dira que ce serait réduire le rôle des chambres à celui des anciens parlements, qui n'avaient que le droit de remontrances, mais qui devaient enregistrer les édits lorsque le roi ordonnait qu'ils le fussent par autorité. Ce raisonnement serait mal fondé car il n'y a aucune parité dans l'espèce ; les édits du conseil étaient jadis l'œuvre non discutée d'un ministre qui souvent pouvait paraitre injuste ou dangereux. Dans la Charte de 1814, ce n'était plus un édit mais une loi, élaborée au conseil d'État, admise par le Conseil des Ministres, sanctionnée par le Roi, enfin approuvée par une des Chambres, ce qui est fort différent d'un édit tout personnel.

Outre ce défaut d'équilibre, on pouvait encore reprocher à ce pacte d'avoir établi la liberté de la presse comme un principe constitutif, car malgré la restriction des lois répressives c'était dans les circonstances orageuses où l'on se trouvait, établir un germe de conflit dangereux et inévitable.

Quoi qu'il en soit de la proposition qui précède, il est certain qu'une charte trop détaillée sera toujours une œuvre fragile, exposée aux déceptions toutes les fois qu'il s'y trouvera une porte ouverte pour qu'une minorité ambitieuse et factieuse puisse battre en brèche l'autorité royale légalement et progressivement.

Les beaux fruits de la Charte de 1814 modifiée en 1830 n'ont-ils pas été une scandaleuse lutte de portefeuilles ministériels entre quatre ou cinq princes de la parole que les Grecs nommaient des Rhéteurs?

Après avoir analysé succinctement les diverses formes de gouvernement aujourd'hui admises en Europe, je me permettrai d'examiner ce que l'on pourrait convenablement en appliquer à la Russie.

Dans l'état où l'affranchissement des serfs a placé l'Empire avec les complications qui résultent de l'annexion de la Pologne et du droit révolutionnaire des nationalités, en présence des immenses réformes que réclament les institutions judiciaires et administratives du pays, on se demande s'il ne serait pas du plus haut intérêt pour le monarque de ne point assumer à lui seul toute la responsabilité du mal qui pourrait se faire et qui lui aliénerait peu à peu l'amour et la confiance de la nation auxquels il a acquis tant de droits, et à cet effet, ne serait-il pas indispensable de s'entourer de tous les conseils qui pourraient l'éclairer, sans recourir aux assemblées délibérantes dont nous avons signalé les dangers.

Si le généreux empereur Alexandre, animé par le même esprit dont les grands législateurs de toutes les époques ont été inspirés, veut consolider et compléter son œuvre immortel de l'émancipation, il semble donc qu'il serait urgent de procéder 
avec sagesse et mesure à la réorganisation des autorités supérieures en adoptant le programme ci-après :

$1^{\circ}$ Indépendamment du Conseil des Ministres institué par l'Oukase du 24 novembre ${ }^{1}$, on pourrait désigner un Conseil privé de la couronne dont les membres sans attributions fixes seraient choisis parmi les plus expérimentés et les plus habiles ne se réunissant que sur l'appel de l'Empereur et dans les circonstances d'une haute importance. (NB. cette institution n'est pas indispensable et ne serait qu'un perfectionnement dans l'intérêt personnel du Monarque).

$2^{\circ}$ Établir un Conseil d'État composé d'hommes spéciaux pour les différentes parties du service public, chargé de la préparation des Décrets Impériaux, oukases ou lois. Ce Conseil comprendrait six sections, la Justice, les Finances et le $\underline{\text { Commerce, }}$ l'administration intérieure, la Guerre, la Marine, chaque section composée de cinq conseillers spéciaux.

$3^{\circ}$ Instituer le plus tôt possible un Conseil législatif mixte, chargé de la discussion des projets de lois qui lui seraient transmis par le Conseil des Ministres, sur l'ordre de l'Empereur. Ce conseil législatif ne pourrait discuter que les projets élaborés au Conseil d'État sur la demande du Conseil des Ministres, il pourrait y proposer des amendements ou des modifications qui seraient soumis à la sanction du ministère impérial.

Pour donner à ce conseil législatif toute l'autorité compatible avec celle du trône, il serait convenable du moins pour le présent, qu'il fût composé d'un nombre égal de conseillers désignés par l'Empereur et d'un pareil nombre de conseillers désignés par la noblesse des provinces, et par les municipalités des principales villes. Par exemple :

54 Conseillers de la noblesse

54 Conseillers choisis dans les commerçants et bourgeois et notables $\underline{108}$ Conseillers choisis par l'Empereur. 216

(On pourrait même porter à 112 le nombre des Conseillers nommés par la couronne en tout 220 , ce qui donnerait une majorité assurée dans tous les cas.)

$4^{\circ}$ Enfin, il faudrait une haute Cour Impériale de Justice formant à la fois une Cour de Cassation pour les jugements civils et une haute Cour criminelle devant servir de cour d'appel pour les jugements criminels des tribunaux de gouvernement et de haute cour, pour juger les crimes contre la Sûreté de l'État et la famille impériale, rôle qui, dans la charte de Louis XVIII était dévolu à la Chambre des pairs.

La grande question si l'on adoptait cette organisation serait d'assigner le rôle qui serait donné à l'avenir au Sénat et au conseil de l'Empire, dont la transformation deviendrait indispensable; la dernière surtout n'offrirait aucune difficulté ; il suffirait de constituer le conseil de l'Empire comme noyau du

1. L'Oukase du 24 novembre 1861 fut, par une circonstance bizarre, promulgué à Saint-Pétersbourg le jour même où je terminais la présente notice à Paris, et où j'insistais sur la prompte institution de ce conseil, sans lequel il ne peut exister aucun ensemble dans l'administration de l'État. 
Conseil législatif, mentionné plus haut: quant au Sénat, il y aurait trois alternatives à choisir :

a) On pourrait en transporter la moitié dans le conseil législatif et transformer l'autre en conseil d'État.

b) On pourrait au contraire diviser le Sénat en deux sections ; l'une formerait le Conseil d'État, l'autre serait destinée à remplir les fonctions de la Haute Cour impériale de justice, mentionnée à l'article 4 ci-dessus.

c) $\mathrm{Si}$, contre mon opinion on trouvait ces dernières fonctions inférieures au rang actuel des sénateurs on pourrait les fusionner entièrement avec le Conseil de l'Empire, dans le nouveau Conseil législatif, sauf à organiser la haute cour de justice et le Conseil d'État avec d'autres fonctionnaires. Mais je ne pense pas que l'on dût en venir là, et il paraît évident que la division du Sénat en deux sections mentionnées à l'article précédent serait la plus rationnelle.

Quoi qu' il en soit, dans tout ce remaniement des hauts fonctionnaires de l'Empire, on rencontrerait une certaine difficulté, assez sérieuse, ce serait l'abolition indispensable des Tchin ou des rangs. Comment en effet pourrait-on donner des masses de titres de Conseillers privés à des employés de finances, de justice, de douane, même quand ce titre devrait être réservé aux hommes d'élites appelés à former réellement le Conseil privé de la Couronne. Il en est de même du titre de Conseiller d'État qui ne saurait s'appliquer comme aujourd'hui à tous les employés publics au bout d'un certain nombre d'années de service et n'appartient en effet qu'aux membres réels du Conseil d’État chargés de la préparation des projets de lois réclamés par le Cabinet impérial à mesure que la marche des affaires l'exige. Pour éviter cette confusion, il suffirait de donner des titres de Conseillers de finances, ou de justice, ou de $1^{\circ}, 2^{\circ}, 3^{\circ}$ classe sans autre désignation. Les employés subalternes conserveraient leurs titres.

La plus grave des questions exposées dans les lignes qui précèdent serait certainement l'établissement d'un Conseil législatif auquel serait adjoint un certain nombre de conseillers élus par la noblesse des 54 gouvernements et par les bourgeois des villes. Cette institution qui serait dégagée de tous les dangers des assemblées délibérantes ambitieuses, aurait le grand avantage de donner la sanction nationale aux mesures du gouvernement, ce qui pour le crédit public et les ressources financières est de la plus haute importance.

J'ai signalé dans les premières pages de ces Rêveries comme dans mon précis politique de la campagne de 1815 , toute la nullité du prétendu équilibre des pouvoirs et les dangers des Chambres disposées à restreindre l'autorité royale, on ne saurait donc m'accuser de vouloir flatter les utopistes libéraux qui ont fait tant de mal en rêvant tant de bien; mais je demeure convaincu que le Conseil législatif mitigé selon l'article 4 susmentionné serait le plus puissant levier que l'autorité impériale pût employer pour détruire les abus de l'administration subalterne et fonder le règne de la justice, sans exposer l'autorité impériale à aucun danger. Je terminerai enfin en proposant l'établissement d'un ministère de la police, afin de régulariser l'action immense que cette autorité peut et doit exercer dans tout l'Empire dans ces années de transition entre le servage et l'émancipation. À cette institution, il faudrait joindre un petit code de police judiciaire bien élucidé. 
On me pardonnera l'outrecuidance de ces propositions rédigées d'abord pour ma propre satisfaction et destinées à une communication confidentielle au plus fidèle des serviteurs de sa Majesté2.

\section{Appendice}

On remarquera que toutes les nouvelles institutions proposées sont basées sur les principes du gouvernement personnel absolu, dont il me paraît qu'il serait imprudent de s'écarter dans les graves circonstances actuelles, sauf à étendre plus tard les attributions des assemblées consultatives.

Après avoir exposé mes idées sur les créations les plus urgentes dans l'état actuel des choses en Russie, on me permettra d'examiner ce qui pourrait surgir plus tard, si sa Majesté jugeait opportun de faire un pas de plus vers la monarchie tempérée, en conservant soigneusement l'initiative et la force indispensables à l'autorité impériale mais en augmentant les moyens d'éclairer sa marche et de la mettre en rapport avec la nation sans s'exposer au risque de l'ébranler.

Sa Majesté pourrait couronner l'édifice qui immortalisera son règne en établissant une Chambre des Pairs nommés par elle à vie et choisis dans la noblesse et dans toutes les classes de la nation parmi les fonctionnaires distingués par leur mérite et leurs services rendus à l'État.

Pour faire apprécier cette institution et en écarter tout ce qui paraîtrait de nature à porter ombrage je me permettrai d'en présenter l'abrégé sous la forme d'un Oukase qui réunirait tous les rouages du gouvernement en admettant dans sa rédaction que le Conseil d'État et le Conseil législatif mixte auraient déjà été antérieurement décrétés et installés.

\section{$\underline{\text { Formule d'un Oukase }}$}

Nous, Alexandre II, Empereur autocrate de toutes les Russies, et voulant donner à Notre Noblesse comme à toutes les classes de nos fidèles sujets un témoignage de notre munificence et de Notre vive sollicitude pour tout ce qui peut contribuer au développement successif et durable de leur prospérité.

Avons ordonné et ordonnons ce qui suit :

$1^{\circ}$ Indépendamment du Conseil législatif mixte, du Conseil d'État et de la haute Cour de justice y établis par notre oukase du (...) en remplacement du Sénat et du Conseil de l'Empire (on suppose ce remplacement déjà effectué) il sera établi une Chambre de pairs selon les règles suivantes ;

$2^{\circ}$ Ces pairs seront nommés par nous à vie en tel nombre que nous le jugerons convenable parmi la noblesse ou parmi les fonctionnaires supérieurs de toutes les classes distingués par leur mérite et par de grands services rendus à l'État, nous

2. J'avais d'abord voulu soumettre ces idées au Comte Kissdef [Kiselev], ambassadeur de sa Majesté à Paris, mais de grandes occupations et les fêtes de la fin de l'année l'ayant empêché de me donner une audience assez longue pour le discuter, je profitais d'un courrier pour les envoyer à mon fils qui les soumit à l'empereur avec une rédaction un peu modifiée. 
réservant par exception d'accorder la pairie héréditaire aux généraux, amiraux, ambassadeurs ou ministres d'État ayant rendu des services signalés à la gloire et à la grandeur de l'Empire ;

$3^{\circ}$ La pairie héréditaire cessera d'avoir son effet avec la $3^{e}$ génération à moins qu'elle ne soit prolongée par l'Empereur dans la personne du $3^{\mathrm{e}}$ titulaire ;

$4^{\circ}$ Dorénavant tous les projets de loi (ou d'oukase) d'abord élaborés et rédigés au Conseil d'État à la requête des ministres et après notre approbation seront présentés en notre nom au Conseil législatif ${ }^{3}$ puis à la chambre des pairs qui pourront y introduire des amendements ou changements de rédaction qu'avec le consentement impérial ;

$5^{\circ}$ Pour le même motif ces deux assemblées législatives ne pourront mettre en délibération que des projets qui leur seront transmis sur l'ordre de l'Empereur par un de ses ministres ;

$6^{\circ}$ Dans le cas où l'une des deux assemblées aurait voté l'adoption du projet de loi tandis que l'autre y trouve des inconvénients de nature à en désirer le rejet, celleci serait autorisée à présenter une respectueuse adresse à l'Empereur pour lui soumettre ses observations, si le ministre impérial après en avoir délibéré et pris les ordres de l'Empereur, persistait dans l'adoption du projet, il serait alors enregistré dans le Code pour avoir force de loi ;

$7^{\circ}$ Aucune de ces assemblées ne pourra se réunir sans y être convoquée par Nous, et ne devra siéger que durant le terme que nous déterminerons chaque année. L'oukase de leur convocation désignera aussi les Président, vice-président et secrétaires de chacune d'elle ;

$8^{\circ}$ Notre Conseil des ministres fera élaborer sans délai par le Conseil d’État, et présentera le plus promptement possible à Notre approbation, les oukases organiques nécessaires pour régler le fonctionnement intérieur de chacune de ces assemblées, leur rapports tant entre elles qu'avec Notre Gouvernement Impérial.

La combinaison mixte que nous venons d'esquisser n'offrirait aucun des dangers du gouvernement parlementaire et présenterait des avantages dont on ne saurait reconnaître l'importance.

Elle donnerait satisfaction aux familles illustres de la Russie en les appelant par la Pairie à la discussion consultative des intérêts de l'Empire.

Elle donnerait satisfaction aux classes moyennes par le même motif.

Elle donnerait plus de solennité aux lois ainsi sanctionnées.

Le crédit public financier s'en trouverait raffermi et multiplié par la suppression des abus.

Enfin, la responsabilité du monarque serait couverte par la discussion loyale des mesures adoptées et son autorité loin d'en être altérée, n'en serait peut-être que plus forte et mieux appréciée. 
On comprend que tout ce qui précède serait la pierre fondamentale sur laquelle le Monarque pourrait élever dans la suite et peu à peu un édifice approprié aux circonstances, aux besoins du pays, au maintien de l'autorité ; un monument enfin qui suffirait à immortaliser tout un règne.

Je me suis appliqué à exposer le pour et le contre avec la ferveur d'un homme convaincu que trop de liberté mène à l'anarchie et à la ruine, comme trop de despotisme mène à la démoralisation des autorités subalternes, à une anarchie aussi funeste que celle d'une liberté excessive, enfin à l'affaissement d'une nation par la compression de tout esprit public.

Je sais que mes idées feront sourire les doctrinaires partisans du self gouvernement anglais et même les libéraux, moins exclusifs. Peu importe, je n'écris pas ces lignes pour obtenir les suffrages mais pour un pays à qui les utopies ne sauraient convenir. Et si, par contre, il existait aussi des partisans outrés de l'absolutisme qui vissent des tendances trop libérales dans l'institution de deux conseils législatifs, malgré les limites restreintes de leurs attributions, je leur citerais l'opinion du roi Guillaume de Hollande. Ce prince si éclairé (malgré les fautes que les Belges lui ont reprochées) me dit en 1827, en parlant des mesures réactionnaires du roi Charles $\mathrm{X}$ : « De quoi se plaint-il ? Il a l'initiative absolue de toutes les lois, la nomination à tous les emplois civils et militaires, un budget de 12 à 1500 millions voté tous les ans sans objections. On voudrait me faire Empereur de Russie que je n'accepterais le fardeau de l'Empire qu'avec les mêmes conditions. » Et cependant les chambres établies par la Charte de Louis XVIII étaient bien autrement puissantes et redoutables que les assemblées que je propose.

Quoi qu'il en soit, je réclame l'indulgence pour ces rêveries d'une vieille tête de 83 ans, au bord du tombeau et qui croirait offrir plus que sa vie au généreux Empereur Alexandre II en lui soumettant ces délicates élucubrations. 
